Document downloaded from:

http://hdl.handle.net/10251/58964

This paper must be cited as:

Jorda Mora, E.; Zarco Garcia, AM. (2014). Weighted Banach spaces of harmonic functions. Revista de la Real Academia de Ciencias Exactas, Fisicas y Naturales. Serie A. Matematicas. 108(2):405-418. doi:10.1007/s13398-012-0109-z.

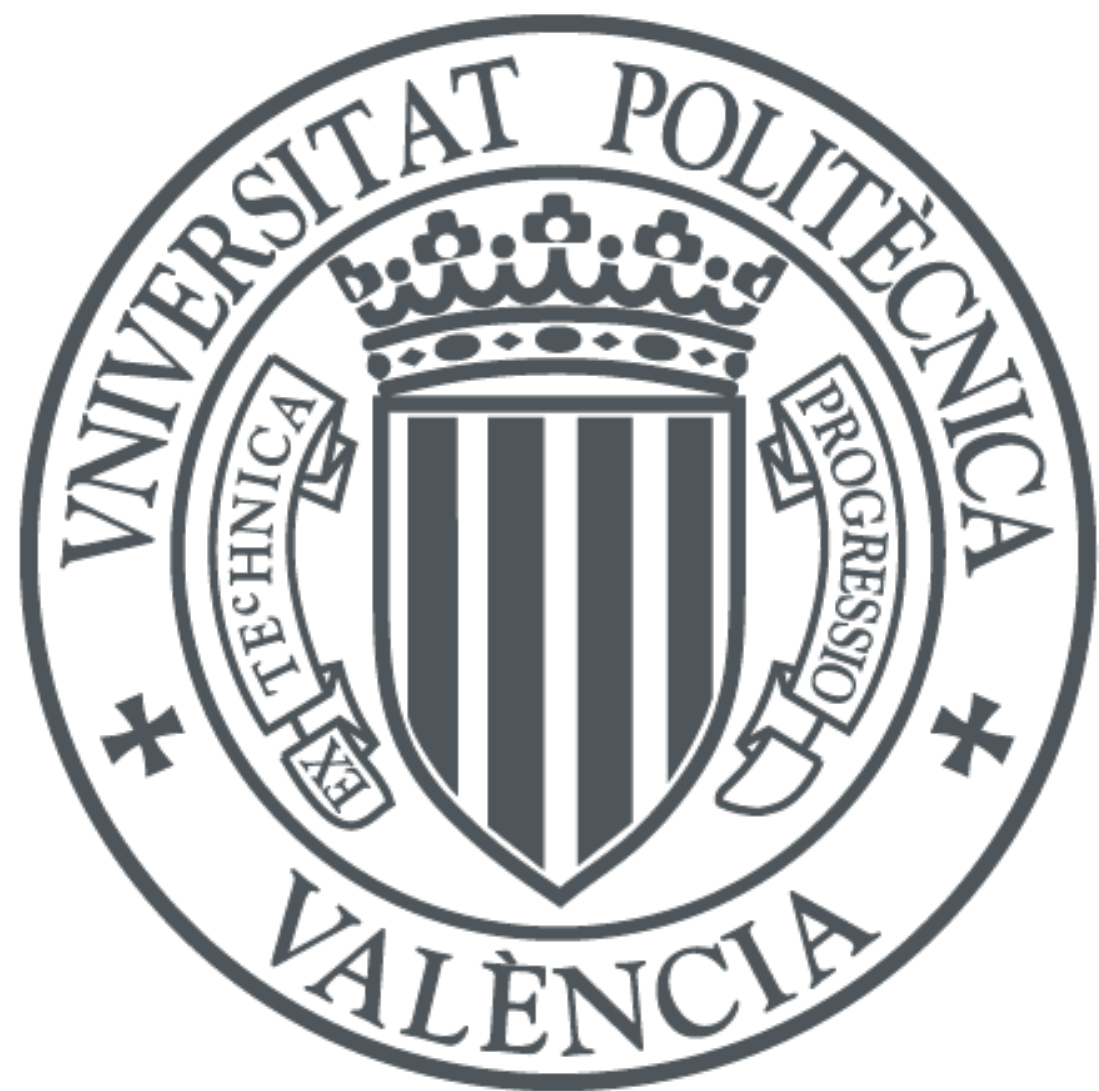

The final publication is available at

http://dx.doi.org/10.1007/s13398-012-0109-z

Copyright Springer Verlag

Additional Information

"The final publication is available at Springer via http://dx.doi.org/10.1007/s13398-012-0109z." 


\title{
Weighted Banach spaces of harmonic functions
}

\author{
Enrique Jordá Mora*, \\ Ana María Zarco García
}

June 21, 2012

\begin{abstract}
We study Banach spaces of harmonic functions on open sets of $\mathbb{R}^{N}$ endowed with weighted supremum norms. We investigate the harmonic associated weight defined naturally as the analogue of the holomorphic associated weight introduced by Biersted, Bonet, and Taskinen and we show that they are not equal in general. We study composition operators with holomorphic symbol between weighted Banach spaces of pluriharmonic functions characterizing the continuity, the compactness and the essential norm of composition operators among these spaces in terms of associated weights.
\end{abstract}

\section{Introduction}

Weighted Banach spaces of holomorphic functions with weighted supremum norms and composition operators between them have been studied by Bierstedt, Bonet, Contreras, Domański, Galbis, García, Hernández-Díaz, Lindström, Maestre, Rueda, Sevilla-Peris and Taskinen and others, see [2, 5, $6,7,13,14,15]$ and the references therein.

Spaces of harmonic functions have been investigated by Shields and Williams [27], in connection with the growth of the harmonic conjugate of a function. In [26], they proved results of duality for weighted spaces of harmonic functions on the open unit disk. Lusky also considers weighted spaces of harmonic functions in $[19,20]$, where the isomorphism classes in the case of radial weights on the disk are determined.

${ }^{*}$ The research of the first author was partially supported by MEC and FEDER, Project MTM2007-62643, and MEC, Project MTM2007-30904-E, and Conselleria d'Educació de la GVA, Ajuda complementaria ACOMP/2009/253. 
In $[9,10,11]$ Boyd and Rueda investigate in the geometry of $h_{v_{0}}(G)$ where $G$ is an open set of the complex plane, examining topics such as the harmonic and holomorphic $v$-boundary and $v$-peak points.

Our aim here is to study conditions making possible to extend some of the known facts in weighted spaces of holomorphic functions to weighted spaces of harmonic functions. First, in the next section, we introduce the harmonic associated weight $\tilde{v}_{h}$ in these spaces in a natural way extending the work on the corresponding spaces of holomorphic functions due to Bierstedt, Bonet and Taskinen in $[3,1 . \mathrm{A}]$. This is a very important tool for the study of these spaces. It is better linked to the space than the original weight, for example it has been used to study the most important properties between composition operators between weighted Banach spaces of holomorphic functions with holomorphic symbol. We extend most of the results in that paper to the harmonic case, and we check that in general the holomorphic and the harmonic associated weight are different. We give also some conditions under which they coincide. In section 3, the composition operators are analyzed on weighted spaces of pluriharmonic functions, which is the convenient context to consider the composition operator with holomorphic symbols. We characterize the norm and the essential norm for some important weights, extending results from $[5,22]$ to these spaces.

\section{Harmonic associated weights.}

Our notation for locally convex spaces and complex and functional analysis is standard. We refer the reader to [16, 17, 18, 21, 24, 25].

Let $G$ be an open and connected set of $\mathbb{R}^{N}$ (or in $\mathbb{C}^{N}$ ). We denote by $h(G)$ the space of complex valued harmonic functions on $G$. If $G \subset \mathbb{C}^{N}$ we denote by $H(G)$ the space of holomorphic functions on $G$. We have the inclusion $H(G) \subseteq h(G)$. We denote by $\|\cdot\|$ the euclidian norm in $\mathbb{R}^{N}$ or in $\mathbb{C}^{N}$ and $|\cdot|$ the modulus of a real or a complex number. Some times we make an abuse of notation and $|\cdot|$ denotes an arbitrary norm in $\mathbb{R}^{N}$ or in $\mathbb{C}^{N}$ and the modulus in $\mathbb{R}$ or $\mathbb{C}$. A weight on $G$ is a function $v: G \rightarrow \mathbb{R}$ which is strictly positive and continuous. Every weight has associated a function $w$ called growth condition defined by $w:=\frac{1}{v}$. For a weight $v$ the weighted Banach spaces of holomorphic functions with weight $v$ are defined by:

$$
\begin{aligned}
h_{v}(G): & =\left\{f \in H(G):\|f\|_{v}:=\sup _{z \in G} v(z)|f(z)|<\infty\right\}, \text { and } \\
h_{v_{0}}(G): & =\{f \in H(G): v f \text { vanishes at infinity on } G\}
\end{aligned}
$$


We say that a function $g: G \rightarrow \mathbb{C}$ vanishes at infinity on $G$ if for each $\varepsilon>0$ there exists a compact set $K \subset G$ such that $|g(z)|<\varepsilon$ for each $z \in G \backslash K$. If $G \subseteq \mathbb{C}^{N}$, the corresponding spaces of holomorphic functions $H_{v}(G)$ and $H_{v_{0}}(G)$ have been deeply studied as we mentioned in the introduction. The unit balls in these spaces are denoted by $b_{v}, b_{v_{0}}, B_{v}$ and $B_{v_{0}}$. The balls $B_{v}$ and $b_{v}$ are compact if we endow them with the compact open topology $\tau_{0}$.

We say that two weights $v_{1}, v_{2}: G \rightarrow \mathbb{R}$ are equivalent if there exist two positive constants $C$ and $D$ such that $C v_{1} \leq v_{2} \leq D v_{1}$ and we write $v_{1} \sim v_{2}$.

An open and connected set (domain) $G$ of $\mathbb{R}^{N}$ (or $\mathbb{C}^{N}$ ) is called balanced if for each $z \in G$ and for each $\lambda \in \mathbb{R}(\lambda \in \mathbb{C})$ with $|\lambda| \leq 1$ we have $\lambda z \in G$. A weight $v: G \rightarrow \mathbb{R}$ on a balanced domain $G$ is radial if $v(z)=v(\lambda z)$ for all $z \in G$ and for all $|\lambda|=1$. It is called unitary for a norm $|\cdot|$ if there exists a positive continuous function $g:[0, \infty[\rightarrow] 0, \infty[$ such that $v(x)=g(|x|)$. We say that $v$ is unitary when it is unitary for the euclidian norm $\|\cdot\|$.

Let $v$ be a weight on $G$ and let $w=1 / v$ be its related growth condition. By $\widetilde{w}_{h},: G \rightarrow \mathbb{R}$ we denote the function

$$
\widetilde{w}_{h}(z):=\sup \left\{|f(z)|: f \in b_{v}\right\},
$$

the harmonic weight associated with $v$ is $\widetilde{v}_{h}:=\frac{1}{\widetilde{w}_{h}}$. For each $z \in G$ we consider the function $\delta_{z}:\left(h(G), \tau_{0}\right) \rightarrow \mathbb{C}$ defined by $\delta_{z}(f):=f(z)$ (here $\tau_{0}$ denotes the compact open topology). This function is called the evaluation at $z$ and it is linear and continuous. The compactness of the unit ball $b_{v}$ in $\left(h(G), \tau_{0}\right)$ implies that the supremum in the definition is a maximum. Since the norm topology on $h_{v}(G)$ is stronger than the one induced by $\tau_{0}$, we have that the restriction $\delta_{z}: h_{v}(G) \rightarrow \mathbb{C}$ is also linear and continuous. We denote by $h_{v}(G)^{\prime}$ the topological dual space of $h_{v}(G)$. From the very definition we have $\widetilde{w}_{h}(z)=\left\|\delta_{z}\right\|_{h_{v}(G)^{\prime}}$. In case $G \subseteq \mathbb{C}^{N}$ the holomorphic associated weight is denoted by $\widetilde{v}_{H}$ and its corresponding associated growth condition by $\widetilde{w}_{H}$.

We present below a list of properties satisfied by the the growth conditions $w, w_{1}$ and $w_{2}$ and the weight $v$ defined on $G$, extending the well known results in the holomorphic case to to the harmonic case.

1. Let $f \in h(G)$. Then $|f| \leq w \Leftrightarrow|f| \leq \widetilde{w}_{h}$. This yields that $h_{v}(G)$ and $h_{\widetilde{v}_{h}}(G)$ are isometric.

2. For each $z_{0} \in G$ there exists $f_{z_{0}} \in h(G)$ such that $\left|f_{z_{0}}(z)\right| \leq w(z)$ for all $z \in G,\left|f_{z_{0}}\left(z_{0}\right)\right|=\widetilde{w}_{h}\left(z_{0}\right)$,

3. $\widetilde{w}_{h}$ is continuous and subharmonic,

4. $w_{1} \leq w_{2} \Rightarrow \widetilde{w}_{1 h} \leq \widetilde{w}_{2 h}$ 
5. $(C w) \widetilde{h}=C \widetilde{w}_{h}$ for every constant $C>0$,

6. $\left(\widetilde{w}_{h}\right) \widetilde{h}=\widetilde{w}_{h}$,

7. $\left(\min \left(w_{1}, w_{2}\right)\right) \widetilde{h}=\left(\min \left(\widetilde{w}_{1 h}, \widetilde{w}_{2 h}\right)\right) \widetilde{h}$,

8. $\left(\max \left(\widetilde{w}_{1 h}, \widetilde{w}_{2 h}\right)\right) \widetilde{h} \leq\left(\max \left(w_{1}, w_{2}\right) \widetilde{h}\right.$.

9. If $G \subseteq \mathbb{C}^{N}$ then $\widetilde{w}_{H} \leq \widetilde{w}_{h} \leq w$.

10. If $G$ is balanced ( $G$ is a ball or $G=\mathbb{R}^{N}$ ) then $\widetilde{w}_{h}$ is radial (unitary) whenever $w$ is.

11. If $\widetilde{w}_{h}$ is unitary in a ball $B$ (or the whole space) for a norm $|\cdot|$ then $\widetilde{w}_{h}$ is non increasing.

12. If $G$ bounded and $v$ is bounded or $G=\mathbb{R}^{n}$ and $v(z)$ tends to zero when $\|z\|$ tends to infinity then $h_{v}(G) \neq 0$, and then $\widetilde{v}_{h}$ is bounded.

13. If $G$ is the unit ball for a norm $|\cdot|$ of $\mathbb{R}^{n}$ or $G=\mathbb{R}^{n}, v$ is unitary for $|\cdot|$ and $h_{v}(G) \neq 0$ then $\sup _{z \in G} v(z)<\infty$. If $G$ is the euclidian ball (or $G=\mathbb{R}^{n}$ ) and $v$ is unitary then $\sup _{z \in G} \widetilde{v}_{h}(z)<\infty$. In case $G=\mathbb{R}^{n}$ we have even $\lim _{|z| \rightarrow \infty} v(z)=0$. If $v$ is unitary with respect to the euclidian norm we also have $\lim _{\|z\| \rightarrow \infty} \widetilde{v}_{h}(z)=0$.

The first 9 properties are extensions to the harmonic case of $[3,1 . \mathrm{A}]$. We do not give the proofs because they are completely analogous. All these properties can be stated in terms of the weights instead of the growth conditions. In particular, 9 will be used during the rest of the paper mainly in the form $v \leq \widetilde{v}_{h} \leq \widetilde{v}_{H}$.

Let prove 10. For each orthogonal transformation $T$ we have that $f \circ T$ is harmonic (cf. [1, Chapter 1]). Moreover, since $v$ is unitary, we have $v(x)|f(T(x))|=v(T(x))|f(T(x))|$ and from this it follows that composition of functions in $h_{v}(G)$ with orthogonal transformations gives an isometry in $h_{v}(G)$. Let $0<r<1$. There exists $f \in h_{v}(G)$ with $\|f\|_{v}=1$ such that $f(r, 0, \ldots, 0)=\widetilde{w}_{h}(r, 0, \ldots, 0)$. Let $x_{0} \in G$ such that $\left\|x_{0}\right\|=r$. If we consider an orthogonal transformation $T$ such that $T\left(x_{0}\right)=(r, 0, \ldots, 0)$. Since $f \circ T$ is in $b_{v}$ we get that $\widetilde{w}_{h}\left(x_{0}\right) \geq f \circ T\left(x_{0}\right)=\widetilde{w}_{h}(r, 0, \ldots, 0)$. If we assume that there is $g \in b_{v}$ such that $g\left(x_{0}\right)>f \circ T\left(x_{0}\right)$, then $g\left(x_{0}\right)=g \circ T^{-1} \circ T\left(x_{0}\right)=g \circ$ $T^{-1}(r, 0, \ldots, 0) \leq w\left(x_{0}\right)=w(r, 0, \ldots, 0)$ and $g \circ T^{-1}$ is in $b_{v}$ because $T^{-1}$ is an orthogonal transformation. This contradicts $f(r, 0, \ldots, 0)=\widetilde{w}_{h}(r, 0, \ldots, 0)$.

To see 11 we consider a weight $v$ on a ball $B$ with respect to a norm $|\cdot|$ which is unitary. Let $0<r_{1}<r_{2}$ and $z_{1} \in B$ with $\left|z_{1}\right|=r_{1}$. We take $f_{0} \in b_{v}$ such that $\left|f_{0}\left(z_{1}\right)\right|=\widetilde{w}_{h}\left(z_{1}\right)$. For the maximum modulus principle 
for harmonic functions $[1,1.8]$ there exists $z_{2}$ such that $\left|f_{0}\left(z_{2}\right)\right| \geq\left|f_{0}\left(z_{1}\right)\right|$. Hence $\widetilde{w}_{h}\left(z_{2}\right)=\sup _{f \in b_{v}}\left|f\left(z_{2}\right)\right| \geq\left|f_{0}\left(z_{1}\right)\right|=\widetilde{w}_{h}\left(z_{1}\right)$. Since $\widetilde{w}_{h}$ is unitary with respect to $|\cdot|$ we conclude. The proof obviously works for the holomorphic case, and it seems that this property 11, despite it is quite obvious, is not written in any paper so far. Properties 10 and 11 together mean that if $v$ is a unitary weight in the unit ball $B$ (or $B$ is the whole space) of $\left(\mathbb{C}^{N},|\cdot|\right)$, then $\widetilde{v}_{h}$ is unitary and non increasing.

The last two properties are extensions of [8, Ob.1]. In both cases in 12 we have $1 \in h_{v}(G)$ and $\widetilde{v}_{h} \leq\|1\|_{v}$.

We see 13 seeking a contradiction, we suppose that $v$ is not bounded. We consider $v(x)=g(|x|)$. Then, it exists a strictly increasing sequence $\left(r_{n}\right)_{n}$ of positive numbers such that $\left(g\left(r_{n}\right)\right)_{n} \rightarrow \infty, r_{n} \rightarrow 1$ when $G$ is the unit ball for $|\cdot|$, and $r_{n} \rightarrow \infty$ when $G=\mathbb{R}^{n}$. From $h_{v}(G) \neq 0$, we get $h_{v}^{\mathbb{R}}(G) \neq 0$. Let $u \in h_{v}^{\mathbb{R}}(G), u \neq 0$ and $M(r)=\sup _{|z|=r}|u(z)|$.

$$
\sup _{n \in \mathbb{N}} g\left(r_{n}\right) M\left(r_{n}\right) \leq \sup _{z \in G} v(z)|u(z)|=\|u\|_{v}<\infty
$$

and furthermore, the sequence $\left(M\left(r_{n}\right)\right)_{n}$ is increasing by the maximum modulus principle of harmonic functions $[1,1.8]$. Hence,

$$
M\left(r_{n}\right) \leq \frac{\|u\|_{v}}{g\left(r_{n}\right)}, \text { for all } n \in \mathbb{N}
$$

Taking limit when $n$ tends to $\infty$ we get a contradiction since $u \neq 0$. If $G=\mathbb{R}^{N}$ and we assume that there exists $c$ and $r_{n} \rightarrow \infty$ such that $v\left(r_{n}\right)>$ $c$, then we obtain that, for each $f \in b_{v}$ and $|z|=r_{n}$ we have $|f(z)| \leq$ $1 / c$. Applying again the Maximum Modulus Principle for harmonic functions we get a contradiction with Liouville Theorem for harmonic functions $[1$, 2.1]. The corresponding statements for $\widetilde{v}_{h}$ when $v=g(\|x\|)$ follow from the equality $h_{v}(G)=h_{\tilde{v}_{h}}(G)$ and the fact that $\widetilde{v}_{h}$ is unitary (property 10 above).

To state the following result, we remark that if $G$ is the unit ball of $\mathbb{R}^{n}$ (or $G=\mathbb{R}^{N}$ ) and $v$ is a unitary weight then Property 10 proved above implies the existence of a positive function $g$ such that $\widetilde{v}_{h}(x)=g(\|x\|)$.

Proposition 2.1. Let $g:[0,1] \rightarrow \mathbb{R}^{+}$(or $g:\left[0, \infty\left[\rightarrow \mathbb{R}^{+}\right.\right.$) be a non increasing positive function and let $G_{n}$ be the unit ball of $\mathbb{R}^{n}$ (or $G_{n}=\mathbb{R}^{n}$ ) for the euclidian norm. Define $v_{n}(x)=g(\|x\|)$ for $x \in \mathbb{R}^{n}$. Let $g_{n}:[0,1] \rightarrow \mathbb{R}^{+}$be the function such that $\left(\widetilde{v}_{n}\right)_{h}(x)=g_{n}(\|x\|)$ for each $n \geq 2$. Then $g_{n+1} \leq g_{n}$ for each $n \geq 2$.

Proof. We only give the proof for $G$ being the unit ball. Let $n \geq 2,0<r<1$. Denote $r_{n}=(r, 0 \ldots, 0) \in \mathbb{R}^{n}$, and let $f$ in the unit ball of $h_{v_{n}}\left(G_{n}\right)$ such that 
$1 / g_{n}(r)=f\left(r_{n}\right)$. Such an $f$ exists because property 2 above. Consider $\tilde{f}\left(x_{1}, \ldots, x_{n}, x_{n+1}\right)=f\left(x_{1}, x_{2}, \ldots, x_{n}\right)$. It is immediate that $\tilde{f} \in b_{v_{n+1}}$. We have $\tilde{f}\left(r_{n+1}\right)=f\left(r_{n}\right)=1 / g_{n}(r) \leq 1 / g_{n+1}(r)$.

Our aim is to connect this harmonic associated weight with the holomorphic one. First we observe that property 9 above is valid for the holomorphic case in the ball (or the whole space) with respect to any norm in $\mathbb{C}^{N}$. Further, Bonet proved in [12, Proposition 2] that, for unitary weights, the corresponding associated weight does not depend neither on the norm nor on the dimension. We state this result below as we need it, the original result is written in terms of entire functions on Banach spaces.

Proposition 2.2 (Bonet). Let $g$ be a positive functions defined on positive numbers. If we consider a norm $|\cdot|$ in $\mathbb{C}^{n}$ and a weight $v=g(|z|)$ for $z \in G_{n} \subset \mathbb{C}^{n}, G_{n}$ being the unit ball of $\mathbb{C}^{n}$ for $|\cdot|$ or $G_{n}=\mathbb{C}^{n}$, then $\widetilde{v}_{H}(z)=\widetilde{g}(|z|)$, where

$$
\widetilde{g}(t)=\left\{\sup |h(z)|: h \in H\left(G_{1}\right)|h(z)| \leq 1 / g(|z|) \text { for all } z \in G_{1},|z|=t\right\},
$$

for $G_{1}=\mathbb{D}$ in case $G_{n}$ the ball of $\left(\mathbb{C}^{n},|\cdot|_{n}\right)$ or $G_{1}=\mathbb{C}$ if we are considering $G_{n}=\mathbb{C}^{n}$.

The argument given there does not work for the harmonic case since $f: \mathbb{C}^{n} \rightarrow \mathbb{C}$ being harmonic does not imply that $\lambda \rightarrow f(\lambda z)(\lambda \in \mathbb{C})$ is harmonic. We look now for conditions which ensure that $\widetilde{v}_{H}=\widetilde{v}_{h}$.

A function $f:] a, b[\subset] 0,+\infty[\rightarrow \mathbb{R}$ is called is called convex in $\log r$ with $r \in] a, b\left[\right.$, when the function $\psi$ defined by $\psi(t):=f\left(e^{t}\right)$ is a convex function and is called log-convex when the function $\psi$ defined by $\psi(t):=\log f\left(e^{t}\right)$, for $t \in \log (] a, b[)$ is a convex function (i.e. $\log f$ is convex in $\log r$ ). The following lemma is [5, Lema 5].

Lemma 2.3 (Bonet,Domański, Lindström). Let $v:[0,1[\rightarrow] 0,+\infty[$ a decreasing and continuous function with $\lim _{r \rightarrow 1^{-}} v(r)=0$. Let $v: \mathbb{D} \rightarrow[0,+\infty[$, and consider the radial extension $(v(z)=v(|z|))$. If $\frac{1}{v}$ is log-convex, then $v$ is equivalent to $\widetilde{v_{H}}$ (and consequently also to $\widetilde{v_{h}}$ )

As a clear consequence we have that for a radial weight $v$ in $\mathbb{D}$ if $\lim _{r \rightarrow 1^{-}} v(r)=$ 0 and $\frac{1}{\widetilde{v}_{h}}$ is log-convex, then $\widetilde{v_{h}}$ is equivalent to $\widetilde{v}_{H}$. In view of $[7$, Proposition 1.1], the hypothesis of $\lim _{r \rightarrow 1^{-}} v(r)=0$ is equivalent to $\lim _{r \rightarrow 1^{-}} \widetilde{v}_{H}(r)=0$. Moreover, because of Proposition 2.2, if $v=g(|z|)$ is equivalent to $\widetilde{v}_{H}$ in $\mathbb{D}$, and we define $v_{n}(z)=g(|z|)$ for $z$ in the unit ball $B_{n}$ of $\left(\mathbb{C}^{n},|\cdot|\right),|\cdot|$ being a norm in $\mathbb{C}^{n}$, Proposition 2.2 implies that there is $\widetilde{g}$ not depending neither on $n$ nor on $|\cdot|$ such that $\left(\widetilde{v}_{n}\right)_{H}(z)=\widetilde{g}(|z|)$. This implies the equivalence 
between $v_{n}$ and $\left(\widetilde{v}_{n}\right)_{H}$ for each $n \in \mathbb{N}$. The order relation $v_{n} \leq\left(\widetilde{v}_{n}\right)_{h} \leq\left(\widetilde{v}_{n}\right)_{H}$ between the associated weights implies that $v_{n}$ and $\left(\widetilde{v}_{n}\right)_{h}$ are also equivalent. It seems remarkable that we do not know if $\left(\widetilde{v}_{n}\right)_{h}$ is unitary with respect to an arbitrary norm $|\cdot|$ if it is not the euclidian.

Although we do not know if for unitary weights in the unit disc the associated and the harmonic weights are equivalent, we have a negative answer for the equivalence of associated weights in the disc with non radial weights. This example is inspired by [3, Proposition 3.6]

Example 2.4. We define the function $g: \partial \mathbb{D} \rightarrow \mathbb{R}$ by: $g\left(e^{i t}\right)=\left|1-e^{i t}\right|^{2}, t \in$ $[-\pi, \pi], g \in L^{1}(\partial \mathbb{D})$, is continuous, $g(1)=0$ and $g \geq 0$. Let $w: \mathbb{D} \rightarrow \mathbb{R}$, The Poisson Kernel of the function $g$, that is, $w(z)=\frac{1}{2 \pi} \int_{-\pi}^{\pi} \operatorname{Re}\left(\frac{e^{i t}+z}{e^{i t}-z}\right) g\left(e^{i t}\right) d t$. By [24, Theorem 11.7], $w$ is harmonic in $\mathbb{D}$. Furthermore, $w$ is positive since $\operatorname{Re}\left(\frac{e^{i t}+z}{e^{i t}-z}\right)=\frac{1-|z|^{2}}{\left|e^{i t}-z\right|^{2}}>0$ and $g>0$ in $\partial \mathbb{D} \backslash\{1\}$. Therefore, $w$ is a growth condition in $\mathbb{D}$ and $\widetilde{w}_{h}=w$.

On other hand, $\log g \in L^{1}(\partial \mathbb{D})$, since

$$
\begin{gathered}
\int_{-\pi}^{\pi} \log \left|1-e^{i t}\right|^{2} d t=\int_{-\pi}^{\pi} \log \left((1-\cos t)^{2}+\sin ^{2}(t)\right) d t= \\
\int_{-\pi}^{\pi} \log (2(1-\cos t)) d t<\infty
\end{gathered}
$$

By applying [3, Corollary 3.7], $\widetilde{w}_{H}=\left|Q_{g}\right|$, where $Q_{g}$ is the outer function of $g$, that is, $Q_{g}(z)=\exp \left(\frac{1}{2 \pi} \int_{-\pi}^{\pi} \frac{e^{i t}+z}{e^{i t}-z} \log g\left(e^{i t}\right) d t\right)$. Since $\log |1-z|^{2}$ is harmonic on $\mathbb{D}$, we conclude $\left|Q_{g}(z)\right|=|1-z|^{2}$.

Let $r \in] 0,1[$,

$$
\begin{gathered}
\frac{\widetilde{v}_{H}(r)}{\widetilde{v}_{h}(r)}=\frac{\widetilde{w}_{h}(r)}{\widetilde{w}_{H}(r)}=\frac{1}{(1-r)^{2}} \frac{1}{2 \pi} \int_{-\pi}^{\pi} \operatorname{Re}\left(\frac{e^{i t}+r}{e^{i t}-r}\right)\left|1-e^{i t}\right|^{2} d t= \\
\frac{1}{(1-r)^{2}} \frac{1}{2 \pi} \int_{-\pi}^{\pi} \frac{1-r^{2}}{\left|e^{i t}-r\right|^{2}}\left|1-e^{i t}\right|^{2} d t \geq \frac{1}{(1-r)} \frac{1}{2 \pi} \int_{-\pi}^{\pi} \frac{1+r}{(1+r)^{2}}\left|1-e^{i t}\right|^{2} d t \geq \\
\geq \frac{1}{1-r^{2}} \frac{1}{2 \pi} \int_{-\pi}^{\pi} 2(1-\cos t) d t=\frac{2}{1-r^{2}}
\end{gathered}
$$

Hence,

$$
\sup _{r \in] 0,1[} \frac{\widetilde{v}_{H}(r)}{\widetilde{v}_{h}(r)}=\infty .
$$

Therefore, the corresponding associated weights $\widetilde{v}_{H}, \widetilde{v}_{h}$ are not equivalent. 
We can also obtain an analogous example with a radial weight considering the punctured disc.

Example 2.5. Let $G=\{z \in \mathbb{C}: 0<|z|<1\}$. Let consider the weight $v$ : $G \rightarrow \mathbb{R}$ defined by $v(z):=\frac{1}{-\log |z|}$, and the corresponding growth condition $w:=\frac{1}{v}$.

Let $f(z)=\log |z|, z \in G$. From $f \in h^{\mathbb{R}}(G)$ and $|f(z)|=w(z)=-\log |z|$ we obtain $\widetilde{w}_{h}=\widetilde{w}_{h}^{\mathbb{R}}=w$.

If $f \in H(G),|f(z)| \leq w(z)$, for all $z \in G$ then $\lim _{z \rightarrow a,|a|=1} f(z)=0$. Moreover $e^{|f(z)|} \leq e^{-\log |z|}=\frac{1}{|z|}$, for all $z \in G$, and then $\left|z e^{f(z)}\right| \leq 1$, for all $z \in G$. This implies that $z e^{f(z)}$ can be holomorphically extended to a function $g$ on $\mathbb{D}$ with $g(z) \neq 0$ if $z \neq 0$. Hence there exists $k \in \mathbb{N}_{0}$ such that $\hat{g}(z):=\frac{g(z)}{z^{k}}$ is holomorphic on $\mathbb{D}$ and satisfies $\hat{g}(0) \neq 0$. Now $\log |\hat{g}|$ is a harmonic function that can be extended continuously to $\partial \mathbb{D}$ as 0 . This yields that $\log |\hat{g}|$ is identically null and consequently $\left|e^{f(z)}\right|=|z|^{k-1}$ for each $z \in \mathbb{D} \backslash\{0\}$. The unique $k \in \mathbb{N}_{0}$ which does not give contradiction is $k=1$. Thus $f$ has to be constantly 0 because of the behaviour when going to the boundary. This means $\widetilde{w}_{H} \equiv 0$.

The following result is inspired in the results of [10, Section 6]. Here, besides putting the more general context, we remove from the weight the condition of being twice differentiable.

Theorem 2.6. Let $g:[0,1] \rightarrow \mathbb{R}_{+}$be a non increasing continuous function such that $g(1)=0$ and $\left.\log \left(\frac{1}{g}\right)\right|_{10,1[}$ is convex. Let $N \geq 2$. Consider the unitary weight $v: B_{\mathbb{R}^{N}} \rightarrow \mathbb{R}_{+}$defined by $v(x):=g(\|x\|)$. Then $v=\widetilde{v}_{h}$. Moreover if $N=2 k$ is even and we consider $v=g(|z|)$ for $|\cdot|$ being a norm in $\mathbb{C}^{k}$ and $z$ in the corresponding unit ball, then we have $v=\widetilde{v}_{H}$.

Proof. We restrict ourselves to the case $v: \mathbb{D} \rightarrow \mathbb{R}^{+}$, considering $v(z)=$ $g(|z|)$. Proving the equality $v=\widetilde{v}_{H}$ in this case, the statement is a consequence of Proposition 2.1, Proposition 2.2 and the order relation $v \leq \widetilde{v}_{h} \leq$ $\widetilde{v}_{H}$.

We fix $r_{0} \in\left[0,1\left[\right.\right.$. Define $\Psi:=\left.\frac{1}{v}\right|_{[0,1[}$. As $\Psi$ is increasing and convex we can get $\alpha_{0} \geq 0$ which depends on $r_{0}$ such that $\Psi(r) \geq \alpha_{0}\left(r-r_{0}\right)+\Psi\left(r_{0}\right)$, for all $r \in[0,1[$. Now we compute

$\begin{aligned} \sup _{0<r<1} v(r) \exp \left(\alpha_{0} r\right) & =\exp \left\{\sup _{0<r<1}\left\{\log v(r)+\alpha_{0} r\right\}\right\} \leq \\ \exp \left\{\sup _{0<r<1}\left\{-\alpha_{0}\left(r-r_{0}\right)-\Psi\left(r_{0}\right)+\alpha_{0} r\right\}\right\} & =v\left(r_{0}\right) \exp \left(\alpha_{0} r_{0}\right)\end{aligned}$ 
Now we consider $f_{0}(z):=\frac{1}{v\left(r_{0}\right) \exp \left(\alpha_{0} r_{0}\right)} \exp \left(\alpha_{0} z\right)$ for $z \in \mathbb{D}$. We have $v\left(r_{0}\right)\left|f_{0}\left(r_{0}\right)\right|=1$

Let $z \in \mathbb{D}$,

$$
v(|z|)\left|f_{0}(z)\right| \leq \frac{v(|z|) \exp \left(\alpha_{0}|z|\right)}{v\left(r_{0}\right) \exp \left(\alpha_{0} r_{0}\right)} \leq 1
$$

and this means $f_{0} \in H_{v}(\mathbb{D})$ and $\left\|f_{0}\right\|_{v}=1$.

Remark 2.7. (a) From the given proof above it follows that for a weight $v(z)=g(|z|)$ defined on $\mathbb{C}$ for $g:\left[0, \infty\left[\rightarrow \mathbb{R}^{+}\right.\right.$continuous and such that $\exp (\alpha z) \in H_{v}(\mathbb{C})$ we have $v=\widetilde{v}_{H}$. This implies that, for such a function $g$ there exists $\widetilde{g}:\left[0, \infty\left[\rightarrow \mathbb{R}^{+}\right.\right.$such that if we define $v_{n}$ in $\mathbb{C}^{n}$ as $v_{n}(z)=g(|z|)$ then $\left(\widetilde{v}_{n}\right)_{H}(z)=\widetilde{g}(|z|)$ for each $n \in \mathbb{N}$ and for each norm $|\cdot|$ defined in $\mathbb{C}^{n}$.

Examples 2.8. In [3, Examples 1.7] and in [10, Example 13] are given weights $v=g(\|z\|)$ defined on the euclidian unit ball of $\mathbb{C}^{n}$ which satisfy that $w=\widetilde{w}_{H}$. We write below some of them for which we have $w=g(|z|)$ with $\log \frac{1}{g}$ convex and $|\cdot|$ being any norm in $\mathbb{C}^{n}$.
(a) $v(z)=\exp \left(C /\left(1-|z|^{\beta}\right), C>0, \beta>1\right.$
(b) $v(z)=(1-|z|)^{\alpha}, \alpha>0$
(c) $v(z)=\arccos (|z|)$,
(d) $v(z)=\cos \left(\frac{\pi}{2}|z|\right)$

\section{Composition operators}

Let $G \subseteq \mathbb{C}^{N}$ be an open and connected subset. A function $f: G \rightarrow \mathbb{C}$ of class $C^{2}$ is said to be pluriharmonic (see for instance $[18,2.2]$ ) if for every complex line $l=\{a+b \lambda\}$ the function $\lambda \rightarrow f(a+b \lambda)$ is harmonic on the set $G_{l} \equiv\{\lambda \in \mathbb{C}: a+b \lambda \in \Omega\}$. This condition is equivalent to:

$$
\frac{\partial^{2} f}{\partial z_{j} \partial \overline{z_{k}}} \equiv 0, \forall j, k=1, \cdots, n
$$

Let $p h(G)$ denote the set of pluriharmonic functions on $G$. In this case we have the inclusions $H(G) \subset p h(G) \subset h(G)[1,16,18]$. If $v$ is a weight on $G$ then we can consider the corresponding weighted Banach space $p h_{v}(G)$, which has a compact unit ball $b_{v}^{p}$ for the compact open topology. We consider also the natural definition for the pluriharmonic associated weight $\widetilde{v}_{p h}$. The inclusions $H_{v}(G) \subseteq p h_{v}(G) \subseteq h_{v}(G)$ imply $v \leq \widetilde{v}_{h} \leq \widetilde{v}_{p h} \leq \widetilde{v}_{H}$. These pluriharmonic associated weights share all the properties of the corresponding 
holomorphic associated weights. Even the argument of Proposition 2.2, which is not valid for the harmonic case, works for the pluriharmonic associated weight.

If $G_{1}$ and $G_{2}$ are open and connected subsets in $\mathbb{C}^{N}$ and $\mathbb{C}^{M}$ and $\varphi$ : $G_{2} \rightarrow G_{1}$ is a holomorphic function then we can consider the composition operator $C_{\varphi}: p h\left(G_{1}\right) \rightarrow p h\left(G_{2}\right), C_{\varphi}(f):=f \circ \varphi$. It is in fact well defined, since if $f \in p h\left(G_{1}\right)$, then $f \circ \varphi \in C^{2}\left(G_{2}\right)$ and $f=u+i v$ with $u, v$ in the space $p h\left(G_{1}\right)^{\mathbb{R}}$ of real valued pluriharmonic functions. By [18, proposition 2.2.3], $u$ and $v$ are locally real parts of holomorphic functions. Now, the composition of holomorphic functions is a holomorphic function (see [16, Theorem 5. Chap 1]). From this, it follows that $u \circ \varphi$ and $v \circ \varphi$ are also locally real parts of holomorphic functions. Thus, by [18, Proposition 2.2.3], $u \circ \varphi, v \circ \varphi \in p h\left(G_{1}\right)^{\mathbb{R}}$. Also, $C_{\varphi}:\left(p h\left(G_{1}\right), \tau_{0}\right) \rightarrow\left(p h\left(G_{2}\right), \tau_{0}\right)$ is a continuous and linear map.

Proposition 3.1. Let $v$ and $w$ be weights on $G_{1}$ and $G_{2}$ respectively. The following conditions are equivalent for the composition operator $C_{\varphi}$ :

(a) $C_{\varphi}: p h_{v}\left(G_{1}\right) \rightarrow p h_{w}\left(G_{2}\right)$ is continuous,

(b) $C_{\varphi}\left(p h_{v}\left(G_{1}\right)\right) \subset p h_{w}\left(G_{2}\right)$,

(c) $\sup _{z \in G_{2}} \frac{w(z)}{\widetilde{v}_{p h}(\varphi(z))}<\infty$,

Moreover, if these equivalences hold then $\left\|C_{\varphi}\right\|=\sup _{z \in G_{2}} \frac{w(z)}{\widetilde{v}_{p h}(\varphi(z))}$.

Proof. The arguments are a simple adaptation of those used in [8] for spaces of holomorphic functions. (a) and (b) are equivalent because of the Closed Graph Theorem. We assume that (a) holds. If (c) does not hold then there exists a sequence $\left(z_{n}\right)_{n} \subseteq G_{2}$ such that $\frac{w\left(z_{n}\right)}{\widetilde{v}_{p h}\left(\varphi\left(z_{n}\right)\right)}$ tends to $\infty$. For each $n \in \mathbb{N}$ we take $f_{n} \in b_{v}^{p}$ such that $f_{n}\left(\varphi\left(z_{n}\right)\right)=\frac{1}{\widetilde{v}_{p h}\left(\varphi\left(z_{n}\right)\right)}$. This implies that, for all $n \in \mathbb{N},\left\|C_{\varphi}\right\| \geq\left|C_{\varphi}\left(f_{n}\right)\right| \geq w\left(z_{n}\right)\left|f\left(\varphi\left(z_{n}\right)\right)\right|=\frac{w\left(z_{n}\right)}{\widetilde{v}_{p h}\left(\varphi\left(z_{n}\right)\right)}$, a contradiction. If (c) is true then for all $f$ in the unit ball of $b_{v}^{p}$ we have $\left\|C_{\varphi}(f)(z)\right\|_{w}=$ $\sup _{z \in G_{2}} w(z)|f \circ \varphi(z)| \leq \sup _{z \in G_{2}} \frac{w(z)}{\widetilde{v}_{p h}(\varphi(z))}\|f\|_{v}$.

To estimate the norm of $C_{\varphi}$ when it is es continuous we proceed in a similar way as in [5] in the holomorphic case, obtaining a slight improvement. From the above argument we have that $\left\|C_{\varphi}\right\| \leq \sup _{z \in G} \frac{w(z)}{\widetilde{v}_{p h}(\varphi(z))}$. The transpose $C_{\varphi}^{t}$ is also continuous and for each $z \in G_{2}$ we have $w(z) \delta_{z} \in b_{w}^{p}$. Now, for each $\left.z \in G_{2},\left\|C_{\varphi}\right\|=\left\|C_{\varphi}^{t}\right\| \geq \| C_{\varphi}^{t}\left(w(z) \delta_{z}\right)\right)\|=w(z)\| \delta_{\varphi(z)} \|=\frac{w(z)}{\widetilde{v}_{p h}(z)}$, when $\|\cdot\|$ denotes both the operator norm and the dual norm.

For essential weights, i.e. those for which $v \sim \widetilde{v}_{H}$, the continuity in the weighted space of holomorphic functions is equivalent to the continuity in the space of pluriharmonic functions For weights on the unit ball of $\mathbb{C}^{N}$ endowed 
with an arbitrary norm $|\cdot|$, defined by $v(x)=g(|x|)$ with $g:] 0,1\left[\rightarrow \mathbb{R}^{+}\right.$being continuous and non increasing this happens when $t \mapsto \log (1 / g)\left(e^{t}\right)$ is convex by [5] and Proposition 2.1.

Given $E$ and $F$ normed spaces, and $A: E \rightarrow F$ a continuous linear map, the essential norm of $A$ is defined by $\|A\|_{e}=\inf \{\|A-K\|: K$ is compact $\}$. It is clear from the definition that $A$ is compact if and only if $\|A\|_{e}=0$. Our purpose in the rest of the section is to extend the results in $[5,22]$ and calculate the essential norm of the composition operator between spaces of pluriharmonic functions.

We present below a generalization of [22, Proposition 2.1] to a wider context. We remark that in the statement $\mathbb{C}^{N}$ could be replaced by $\mathbb{R}^{N}$, but we have preferred to restrict to the complex variables case because our natural examples of spaces of functions satisfying the hypothesis are the spaces of pluriharmonic and holomorphic functions.

Proposition 3.2. Let $G$ be a balanced and connected open subset of $\mathbb{C}^{N}$ and let $v$ be a weight on $G$ which vanishes at infinity and such that there exists $M>0$ such that

$$
\sup _{z \in G, 0<r<1} \frac{v(z)}{v(r z)} \leq M
$$

Then there exists a sequence of operators $\left(T_{n}\right)$ on $\left(h(G), \tau_{0}\right)$ such that $T_{n}$ : $h_{v}(G) \mapsto h_{v}(G)$ is compact for each $n \in \mathbb{N}$ and the following conditions are fulfilled:

(i) $H(G)$ and $p h(G)$ are invariant subspaces of $T_{n}$ for each $n \in \mathbb{N}$

(ii) $\tau_{0}-\lim _{n \rightarrow \infty} T_{n}=I$

(iii) $\limsup _{n \rightarrow \infty}\left\|I-T_{n}\right\| \leq 1$.

Proof. We assume without loss of generality $v(x) \leq 1$ for all $x \in G$. The same proof of Proposition 3.1 shows that the hypothesis on the weight is equivalent to the existence of an $M>0$ such that $\left\|C_{r}\right\| \leq M$ for each $0<r<1$, the norm of the operators taken in $L\left(h_{v}(G)\right)$. For each $0<r<1$ the operator $C_{r}(f)=f(r z)$ is compact on $h_{v}(G)$. This can be checked observing that the image of a bounded sequence pointwise (compact open) convergent to zero is norm convergent to zero. This compactness and the fact that $C_{r}\left(h_{v}(G)\right) \subseteq h_{v_{0}}(G)$ implies that for each $0<r<1$ there exists $L \subset G$ compact such that $v(z)\left|C_{r}(f)(z)\right|<\varepsilon$ for each for all $z \in G \backslash L$ and for all $f \in b_{v}$. The standard compactness argument necessary to prove this is 
the same used in [22, Lemma 2.1] for the space of one variable holomorphic functions on the unit disc and a radial weight on it. Moreover, since $G$ has a fundamental sequence of compact sets which are balanced, it follows that $\tau_{0}-\lim _{n} C_{r_{n}}(f)=f$ for each $f \in h(G)$ and for each sequence $\left.\left(r_{n}\right)_{n} \subset\right] 0,1[$ tending to 1 . Hence the sequence $\left(C_{r_{n}}\right)_{n}$ tends uniformly on $b_{v}$ to the identity for the compact open topology [21, Proposition 23.27], since this subset is relatively compact in this topology.

Let $\left(\varepsilon_{n}\right)_{n}$ be a decreasing sequence of positive numbers tending to zero. These facts above permit us to choose an increasing sequence of positive numbers $\left(r_{n}\right)_{n}$ tending to 1 and a fundamental sequence $\left(L_{n}\right)_{n}$ of compact subsets of $G$ such that

$$
\sup _{f \in b_{v}, z \in L_{n}}\left|\left(I-C_{r_{n}}\right)(f)(z)\right| \leq \varepsilon_{n}
$$

and

$$
\sup _{f \in b_{v}, z \in G \backslash L_{n+1}} v(z)\left|C_{r_{n}} f(z)\right| \leq \varepsilon_{n} .
$$

For each $n \in \mathbb{N}$ we choose $m(n) \in \mathbb{N}$ satisfying $(1+M) / m(n)<\varepsilon_{n}$. We define

$$
T_{n}=\frac{1}{m(n)} \sum_{j=n}^{n+m(n)} C_{r_{j}}
$$

The construction implies that $\left\|T_{n}\right\| \leq M$ for each $n \in \mathbb{N}$ and $\left(T_{n}\right)_{n}$ is a sequence convergent to the identity for $\tau_{0}$. We observe

$$
I-T_{n}=\frac{1}{m(n)} \sum_{j=n}^{n+m(n)}\left(I-C_{r_{j}}\right) .
$$

for $n \leq k \leq n+m(n)$. For $z \in G$, let $j_{0} \geq n$ the minimum such that $z \in L_{j_{0}}$. Then, for each $f \in b_{v}$ we compute

(a) $v(z)\left|f(z)-C_{r_{j}}(f)(z)\right| \leq\left|f(z)-C_{r_{j}}(f)(z)\right| \leq \varepsilon_{j}$ for $j_{0} \leq j$.

(b) $v(z)\left|f(z)-C_{r_{j_{0}-1}}(f)(z)\right| \leq\left\|I-C_{r_{j_{0}}}\right\| \leq 1+M$.

(c) $v(z)\left|f(z)-C_{r_{j}} f(z)\right| \leq 1+\sup _{z \in G \backslash L_{j+1}} v(z)\left|C_{r_{j}} f(z)\right| \leq 1+\varepsilon_{j}$ if $n \leq j \leq$ $j_{0}-2$.

Altogether gives 


$$
\left\|I-T_{n}\right\| \leq \frac{m(n)-1}{m(n)}\left(1+\varepsilon_{n}\right)+\frac{1+M}{m(n)} \leq 1+2 \varepsilon_{n},
$$

which clearly implies (ii).

The next results extend the main theorems in $[5,22]$ to our context.

Theorem 3.3. Let $G_{1} \subset \mathbb{C}^{N}$ be a balanced and connected open subset of $\mathbb{C}^{N}$ and let $v$ be a weight on $G$ which vanishes at infinity and such that there exists $M>0$ such that $\sup _{z \in G, 0<r<1} \frac{v(z)}{v(r z)} \leq 1$. Let $G_{2} \subset \mathbb{C}^{M}$ a connected and open set, let be $w_{2}$ a weight on $G_{2}$ and let $\varphi: G_{2} \rightarrow G_{1}$ be a holomorphic function. For the composition operator $C_{\varphi}: p h_{v}\left(G_{1}\right) \rightarrow p h_{w}\left(G_{2}\right)$ and for any fundamental sequence $\left(K_{n}\right)_{n}$ of compact subsets of $G_{1}$ we have

(a) it holds

$$
\left\|C_{\varphi}\right\|_{e} \leq \lim _{n \rightarrow \infty} \sup _{\varphi(z) \in G_{1} \backslash K_{n}} \frac{w(z)}{\widetilde{v}(\varphi(z))}
$$

(b) $C_{\varphi}$ is compact if and only if $\left\|C_{\varphi}\right\|_{e}=\lim _{n \rightarrow \infty} \sup _{\varphi(z) \in G_{1} \backslash K_{n}} \frac{w(z)}{\widetilde{v}_{p h}(\varphi(z))}=0$.

The same result is true for the corresponding operator considered between the spaces of holomorphic functions.

Proof. To prove (a) we get a sequence $\left(T_{n}\right)_{n}$ of compact operators defined by Proposition 3.2. The invariance of $p h\left(G_{1}\right)$ and $T_{n}\left(h_{v}\left(G_{1}\right)\right) \subseteq h_{v}\left(G_{1}\right)$ implies that $\left.T_{n}\right|_{p h_{v}\left(G_{1}\right)} \in K\left(p h_{v}\left(G_{1}\right)\right)$ for each $n \in \mathbb{N}$. Therefore, $C_{\varphi} \circ T_{n}$ (we are denoting by $T_{n}$ the restriction) is also compact for each $n$. Thus,

$$
\left\|C_{\varphi}\right\|_{e} \leq\left\|C_{\varphi}-C_{\varphi}\left(T_{n}\right)\right\|=\left\|C_{\varphi}\left(I-T_{n}\right)\right\| .
$$

On the other hand, we have that for each $j \in \mathbb{N}$ :

$$
\begin{aligned}
& \left\|C_{\varphi}\left(I-T_{n}\right)\right\|=\sup _{f \in b_{v}^{p}}\left\|C_{\varphi}\left(I-T_{n}\right) f\right\|=\sup _{f \in b_{v}^{p}} \sup _{z \in G_{2}} w(z)\left|\left(I-T_{n}\right) f(\varphi(z))\right| \leq \\
& \leq \sup _{f \in b_{v}^{p}} \sup _{\varphi(z) \in K_{j}} w(z)\left|\left(I-T_{n}\right) f(\varphi(z))\right|+\sup _{f \in b_{v}^{p}} \sup _{\varphi(z) \in G_{1} \backslash K_{j}} w(z)\left|\left(I-T_{n}\right) f(\varphi(z))\right|
\end{aligned}
$$

The first term above goes to 0 as $n$ tends to $\infty$ because $w$ is bounded, $b_{v}^{p}$ is $\tau_{0}$-compact and $T_{n}-I$ is $\tau_{0}$ convergent to 0 [21, Proposition 23.27]. For the second we have the estimate 


$$
\sup _{f \in b_{v}^{p}} \sup _{\varphi(z) \in G_{1} \backslash K_{j}} w(z)\left|\left(I-T_{n}\right) f(\varphi(z))\right| \leq \sup _{\varphi(z) \in G_{1} \backslash K_{j}} \frac{w(z)}{\widetilde{v_{p h}}(\varphi(z))}\left\|I-T_{n}\right\| .
$$

Hence we conclude taking limit when $n$ goes to infinity and from the fact that the inequality is valid for each $j \in \mathbb{N}$.

To show (b) we assume that there is $c>0$ and a sequence $\left(z_{j}\right)_{j}$ such that $\varphi\left(z_{j}\right) \in G_{1} \backslash K_{j}$ and such that $\frac{w\left(z_{j}\right)}{\widehat{v_{p h}}\left(\varphi\left(z_{j}\right)\right)} \geq c$. Since $C_{r}(f) \rightarrow f$ pointwise as $r \rightarrow 1$ and $\left\|C_{r}\right\| \leq M$ by hypothesis, taking a subsequence of $\left(z_{j}\right)_{j}$ if necessary we can get a sequence $\left(f_{j}\right)_{j} \in M b_{v}^{p} \cap p h_{v_{0}}\left(G_{1}\right)$ such that $\left|\widetilde{v}_{p h}\left(z_{j}\right) f_{j}\left(z_{j}\right)-1\right| \leq 1 / 4$ and $v\left(\varphi\left(z_{k}\right)\right)\left|f_{j}\left(\varphi\left(z_{k}\right)\right)\right|<1 / 4$ for each $k>j$. This last condition is possible since each $f_{j}$ is in $h_{v_{0}}\left(G_{1}\right)$ and $\left(\varphi\left(z_{j}\right)\right)_{j}$ tends to infinity in the Alexandroff compactification of $G_{1}$. This yields that $\left(f_{j}\right)_{j}$ is a bounded sequence in $p h_{v}\left(G_{1}\right)$ such that $\left\|C_{\varphi}\left(f_{j}\right)-C_{\varphi}\left(f_{k}\right)\right\| \geq 1 / 2$ for $j \neq k$, an then $C_{\varphi}$ is not compact.

Theorem 3.4. Let $B$ be the unit ball of $\left(\mathbb{C}^{N},|\cdot|\right)$ and let $\varphi: G \rightarrow B$ a holomorphic function on an open and connected set $G$ in $\mathbb{C}^{M}$. Let $g$ : $[0,1] \rightarrow \mathbb{R}^{+}$be a continuous function with $g(1)=0$ and let $v(z)=g(|z|)$ be weight on $B$ such that $\widetilde{v}_{H}=\widetilde{v}_{p h}$. Let $w$ a weight on $G$ which vanishes at $\infty$. Suppose that the operator $C_{\varphi}: p h_{v}(B) \rightarrow p h_{w}(G)$ is continuous. Then

$$
\left\|C_{\varphi}\right\|_{e}=\limsup _{|\varphi(z)| \rightarrow 1} \frac{w(z)}{\widetilde{v}(\varphi(z))}
$$

where $\widetilde{v}$ denotes the common associated weight for the spaces of holomorphic and pluriharmonic functions.

Proof. Because of Theorem 3.3 we only have to show the lower bound of the essential norm. Let consider a compact operator $K: p h_{v}(B) \rightarrow p h_{w}(G)$. We show that the restriction $C_{\varphi}-K$ to $H_{v}(B)$ has norm not smaller than $\lim \sup _{|\varphi(z)| \rightarrow 1} \frac{w(z)}{\widetilde{v}(\varphi(z))}$, concluding from this that the norm of the operator $C_{\varphi}-K$ on $p h_{v}(B)$ has the same lower bound.

We can find a sequence $\left(z_{n}\right)_{n}$ in $G$ with $\left|\varphi\left(z_{n}\right)\right|>1-1 / n$ and

$$
\lim _{n} \frac{w\left(z_{n}\right)}{\widetilde{v}\left(\varphi\left(z_{n}\right)\right)}=\limsup _{|\varphi(z)| \rightarrow 1} \frac{w(z)}{\widetilde{v}(\varphi(z))}
$$

Now, taking a subsequence if necessary, we can assume that there is $a$ in such that $|a|=1$ and $\lim \varphi\left(z_{n}\right)=a$. We apply Hahn Banach Theorem to 
get $b \in \mathbb{C}^{N}$ satisfying $\langle a, b\rangle=1$ and $|\langle x, b\rangle| \leq|x|$ for each $x \in \mathbb{C}^{N}$. We take a sequence $(\alpha(n))_{n}$ of natural numbers tending to $\infty$ such that

$$
\lim _{n \rightarrow \infty}\left\langle\varphi\left(z_{n}\right), b\right\rangle^{\alpha(n)}=1
$$

Let $\left(\varepsilon_{n}\right)_{n}$ be a decreasing sequence of positive numbers tending to 0 . From $B_{v}=\bar{B}_{v_{0}} \tau_{0}[4$, Example 2.1 (ii)], it follows that for each $n \in \mathbb{N}$, we can find $f_{n} \in B_{v_{0}}$ such that

$$
\left|f_{n}\left(\varphi\left(z_{n}\right)\right)\right| \geq \frac{1}{\widetilde{v}\left(\varphi\left(z_{n}\right)\right)}-\varepsilon_{n}
$$

We take $h_{n}(z):=\langle z, b\rangle^{\alpha(n)} f_{n}(z), z \in B$. The sequence $\left(h_{n}\right)_{n}$ is in the unit ball of $H_{v_{0}}(B)$ and converges to 0 in $\tau_{0}$. As we have $H_{v_{0}}^{\prime \prime}(B)=H_{v}(B)$ as a consequence of [4, Corollary 1.2, Example 2.1 (ii)], $\mathrm{Ng}$ construction of the predual of $H_{v}(B)$ [23] implies that the compact open (pointwise) topology $\tau_{0}$ agrees with the weak ${ }^{*}$ topology on $B_{v_{0}} \subset B_{v}$, since it is Hausdorff. Then the pointwise convergence of $\left(h_{n}\right)_{n}$ implies the weak convergence of this sequence in $H_{v_{0}}(B)$. Therefore, since $K$ is a compact operator, it follows

$$
\lim _{n \rightarrow \infty}\left\|K\left(h_{n}\right)\right\|_{w}=0
$$

Now we compute

$$
\begin{gathered}
\left\|C_{\varphi}\left(h_{n}\right)-K\left(h_{n}\right)\right\|_{w} \geq\left\|h_{n} \circ \varphi\right\|_{w}-\left\|K\left(h_{n}\right)\right\|_{w}=\sup _{z \in G} w(z)\left|h_{n}(\varphi(z))\right|-\left\|K\left(h_{n}\right)\right\|_{w} \geq \\
\geq \frac{w\left(z_{n}\right)}{\widetilde{v}\left(\varphi\left(z_{n}\right)\right)}\left|\left\langle\varphi\left(z_{n}\right), b\right\rangle\right|^{\alpha(n)}-w\left(z_{n}\right)\left|T\left(\varphi\left(z_{n}\right)\right)\right|^{\alpha(n)} \varepsilon_{n}-\left\|K\left(h_{n}\right)\right\|_{w}
\end{gathered}
$$

We take limit when $n$ tends to $\infty$ to get the desired inequality.

Concluding remarks. (a) The hypothesis of the above theorem are satisfied by every weight $v=g(|z|)$ for $g:[0,1] \rightarrow \mathbb{R}^{+}$continuous with $g(1)=0$ and $\log (1 / g)$ convex in $(0,1)$ because of Theorem 2.6.

(b) If $v=g(|z|)$ with $g$ no increasing and $g(1)=0$ and no additional assumptions, then Proposition 3.2 can be applied for $G=H_{v}(B)$. For $\varphi: G \rightarrow B$ holomorphic and $w$ being a weight on $B$ vanishing at infinity, if we consider the composition operator $C_{\varphi}: H_{v}(B) \rightarrow H_{w}(G)$ then the proof of Theorem 3.4 shows

$$
\left\|C_{\varphi}\right\|_{e}=\limsup _{|\varphi(z)| \rightarrow 1} \frac{w(z)}{\widetilde{v}_{H}(\varphi(z))}
$$


(c) In the same situation, if we consider $C_{\varphi}: p h_{v}(B) \rightarrow p h_{w}(G)$, then we have

$$
\limsup _{|\varphi(z)| \rightarrow 1} \frac{w(z)}{\widetilde{v}_{H}(\varphi(z))} \leq\left\|C_{\varphi}\right\|_{e} \leq \limsup _{|\varphi(z)| \rightarrow 1} \frac{w(z)}{\widetilde{v}_{p h}(\varphi(z))} .
$$

(d) Zheng proved in [28] that for an inner function $\varphi: \mathbb{D} \rightarrow \mathbb{D}$, the essential norm of the composition operator $C_{\varphi}: H^{\infty}(\mathbb{D}) \rightarrow H^{\infty}(\mathbb{D})$ is either 0 or 1 . In the weighted Banach spaces of holomorphic and harmonic functions the situation differs. Consider the typical weight $v(z)=1-|z|$ in $\mathbb{D}$ and the family of symbols $\varphi_{a, n}(z)=z^{n}\left(\frac{z+a}{1+a}\right), n \in \mathbb{N}, a \geq 0$. Since $\varphi_{a, n}(0)=0$ each $\varphi_{a, n}$ is contractive because of Schwarz lemma, we have

$$
\left\|C_{\varphi_{a, n}}\right\|=\sup _{z \in \mathbb{D}} \frac{v(z)}{v\left(\varphi_{a, n}(z)\right)}=1
$$

where the maximum is attained at $z=0$. For calculating the essential norm, we observe that $\left|\varphi_{a, n}(z)\right| \leq \varphi_{a, n}(|z|)$ to compute

$$
\left\|C_{\varphi_{a, n}}\right\|_{e}=\limsup _{\left|\varphi_{a, n}(z)\right| \rightarrow 1} \frac{v(z)}{v\left(\varphi_{a, n}(z)\right)}=\lim _{r \rightarrow 1^{-}} \frac{1-r}{1-\varphi_{a, n}(r)}=\frac{1+a}{1+n+a n} .
$$

All the values in $] 0,1]$ are attained considering all $a \geq 0$ and all $n \in \mathbb{N}$.

Acknowledgement The authors are grateful to J. Bonet for several discussions and suggestions about this paper.

\section{References}

[1] S. Axler, P. Bourdon, and W. Ramey. Harmonic Function Theory, 2Ed. Springer, 2001.

[2] K. D. Bierstedt, J. Bonet, and A. Galbis. Weighted spaces of holomorphic functions on balanced domains. Michigan Math. J., 40 (2):271-297, 1993.

[3] K. D. Bierstedt, J. Bonet, and J. Taskinen. Associated weights and spaces of holomorphic functions. Studia Math., 127 (2):70-79, 1998.

[4] K. D. Bierstedt and W. H. Summers. Biduals of weighted Banach spaces of analytic functions. J. Austral. Math. Soc. Ser. A, 54(1):70-79, 1993.

[5] J. Bonet, P. Domański, and M. Lindström. Essential norm and weak compactness of composition operators on weighted banach spaces of analytic functions. Canad. Math. Bull, 42 (2):139-138, 1999. 
[6] J. Bonet, P. Domański, and M. Lindström. Weakly compact composition operators on weighted vector-valued banach spaces of analytic mappings. Ann. Acad. Sci. Fenn. Ser A. I. Math., 26:233-248, 2001.

[7] J. Bonet, P. Domański, M. Lindström, and J. Taskinen. Composition operators between weighted banach spaces of analytic functions. Austral. Math. Soc. (Series A), 64:101-118, 1998.

[8] J. Bonet, M. Friz, and E. Jordá. Composition operators between weighted inductive limits of spaces of holomorphic functions. Publ.Math Debrecen (Series A), 67:333-348, 2005.

[9] C. Boyd and P. Rueda. The v-boundary of weighted spaces of holomorphic functions. Ann. Acad. Sci. Math. Fenn., 30:337-352, 2005.

[10] C. Boyd and P. Rueda. Complete weights and v-peak points of spaces of weighted holomorphic functions. Israel J. Math., 155:57-80, 2006.

[11] C. Boyd and P. Rueda. Isometries of weighted spaces of harmonic functions. Potential Analysis, 29 (1):37-48, 2008.

[12] Daniel Carando and Pablo Sevilla-Peris. Spectra of weighted algebras of holomorphic functions. Math. Z., 263(4):887-902, 2009.

[13] M. D. Contreras and G. Hernández-Díaz. Weighted composition operators in weighted banach spaces of analytic functions. J. austral. Math. Soc. (Serie A), 69 (1):41-60, 2000.

[14] D. García, M. Maestre, and P. Rueda. Weighted spaces of holomorphic functions on banach spaces. Studia Mathematica, 138, (1), 2000.

[15] D. García, M. Maestre, and P. Sevilla-Peris. Composition operators between weighted spaces of holomorphic functions on banach spaces. Annales Academiae Scientiarum Fennicae Mathematica, 29:81-98, 2004.

[16] R. Gunning and H. Rossi. Analytic Functions of several complex variables. AMS Chelsea Publising, Providence, Rhode Island, 2009.

[17] K. Hoffman. Banach spaces of Analytic Functions. Prentice-Hall, INC, Englewood Cliffs, N.J, 1962.

[18] Steven G. Krantz. Function theory of several complex variables. AMS, 2001. 
[19] W. Lusky. On weighted spaces of harmonic and holomorphic functions. J. London Math. Soc., 59:309-320, 1995.

[20] W. Lusky. On the isomorphism classes of weighted spaces of harmonic and holomorphic functions. Studia Math., 175 (1):19-45, 2006.

[21] R. Meise and D. Vogt. Introdution to Functional Analysis. Oxford University Press, 1997.

[22] A. Montes-Rodríguez. Weight composition operators on weighted banach spaces of analytic functions. J. London Math. Soc., 61 (2):872-884, 2000 .

[23] Kung Fu Ng. On a theorem of Dixmier. Math. Scand., 29:279-280 (1972), 1971.

[24] W. Rudin. Real and Complex Analysis. MacGraw-Hill, 1970.

[25] W. Rudin. Análisis funcional. Reverté, 1979.

[26] A. L. Shields and D. L. Williams. Bounded projections, duality and multipliers in spaces of harmonic functions. J. Reine Angew. Math., 299/300:256-279, 1978.

[27] A. L. Shields and D. L. Williams. Bounded projections and the growth of harmonic conjugates in the unit disc. Michigan Math. J., 29:3-25, 1982 .

[28] Lixin Zheng. The essential norms and spectra of composition operators on $H^{\infty}$. Pacific J. Math., 203(2):503-510, 2002. 